\title{
Undergraduate elective on optoelectronic materials and devices
}

S. M. Lord

S. M. Lord, "Undergraduate elective on optoelectronic materials and devices," Proc. SPIE 9663, Eighth International Topical Meeting on Education and Training in Optics and Photonics, 96632K (6 October 2003); doi: 10.1117/12.2207470

SPIE Event: Eighth International Topical Meeting on Education and Training in Optics and Photonics, 2003, Tucson, Arizona, United States 


\title{
Undergraduate Elective on Optoelectronic Materials and Devices
}

\author{
S. M. Lord \\ Department of Engineering, University of San Diego, 5998 Alcala Park, San Diego, CA 92110 \\ (619) 260 4507, (619) 2602303 (fax), slord@sandiego.edu
}

\begin{abstract}
An elective course on optoelectronic materials and devices offered at the University of San Diego is described. Main topics include band structure, semiconductor alloys, optical processes, photodetectors, light emitting diodes, laser diodes, fiber optics, and quantum wells. Laboratory projects and innovative pedagogical aspects of the course are also discussed. (C)2003 Optical Society of America OCIS codes: (000.2060) Education, (040.2020) Diode lasers, (040.5160) Photodetectors, (060.2310) Fiber Optics
\end{abstract}

\begin{abstract}
Introduction
From DVD players to fiber optic communication networks, optoelectronics are everywhere. To educate productive modern engineers, it is essential to introduce them to the important concepts underlying the multidisciplinary field of optoelectronics. The importance of this has been discussed for over a decade. In 1992 in a guest editorial, W. T. Cathey said "it seems inevitable that the key technologies for transmitting and perhaps processing information will be based on the manipulation of photons, rather than electrons." [1] Many excellent examples of optoelectronics education for undergraduates have been reported including for example those in a special issue of the IEEE Transactions on Education [2] on optoelectronics education and the previous Education and Training in Optics and Photonics (ETOP) conferences [3].

This paper discusses an elective course in Optoelectronic Materials and Devices which is being developed at the University of San Diego (USD) for juniors and seniors in electrical engineering. The course aims to provide an introduction to optoelectronic materials and devices and to aid the students' professional development by addressing issues such as the ability to critically evaluate technical papers, conduct effective literature research, and express information orally and in writing. Thus in addition to lecture and laboratory, several other opportunities were provided to directly address these professional development issues.
\end{abstract}

\section{Course Structure}

The elective course, EEE 194 Optoelectronic Materials and Devices, consists of three hours of lecture and three hours of laboratory per week. Due to the limited availability of laboratory facilities, the enrollment is capped at eight students. Typically there are about twenty students in junior and senior level required electrical engineering classes at USD. Students usually have a choice of two or three electives in a given semester. In Fall 1999 during its first offering, seven students including juniors and seniors took the course. In Fall 2003, eight seniors took the course. This paper will focus on the 2003 offering. There was one midterm exam and a cumulative final exam. Before each exam, the instructor provided detailed objectives. On the syllabus, the overall course objectives were stated as

By the end of the course, students should be able to:

1. Describe, using band diagrams and appropriate equations, the physical operation of important optoelectronic devices including semiconductor lasers, light emitting diodes (LEDs), and photodetectors.

2. Identify key performance parameters of lasers, LEDs, and photodetectors and be able to calculate or design to achieve these parameters using appropriate data, information, and equations.

3. Explain the materials issues involved in the design of lasers, LEDs, and photodetectors.

4. Describe typical characterization techniques for optoelectronic materials and devices including equipment required, information obtained, and performance parameters.

5. Conduct laboratory experiments to characterize optoelectronic sources and detectors.

6. Identify at least 2 current topics in optoelectronics research.

7. Conduct effective literature research including critically evaluating technical papers, and expressing information orally and in writing.

Note that these objectives are also designed to satisfy ABET 2000's criteria a through k. 
Active learning was emphasized in this course beginning with the syllabus where the instructor explained that her class philosophy is based on research which has demonstrated that the best learning occurs when the learner is actively involved. Thus students are expected to come to class on time prepared to think, participate, and learn. Lecture notes are prepared using a word processor and distributed to the students. Typically some sections are left for students to fill in including answers to simple questions and working out of example problems. Students may be called upon individually to answer questions during lecture and usually work on problems in small groups. Throughout the lecture notes, attempts were made to appeal to different learning styles including having many figures for visual learners (see for example Figure 1), text for verbal learners, an organized outline for sequential learners, emphasis on motivation for global learners, applications for intuitive learners, specific examples for sensing learners, problem solving for active learners, and sufficient detail for future review for reflective learners [4].

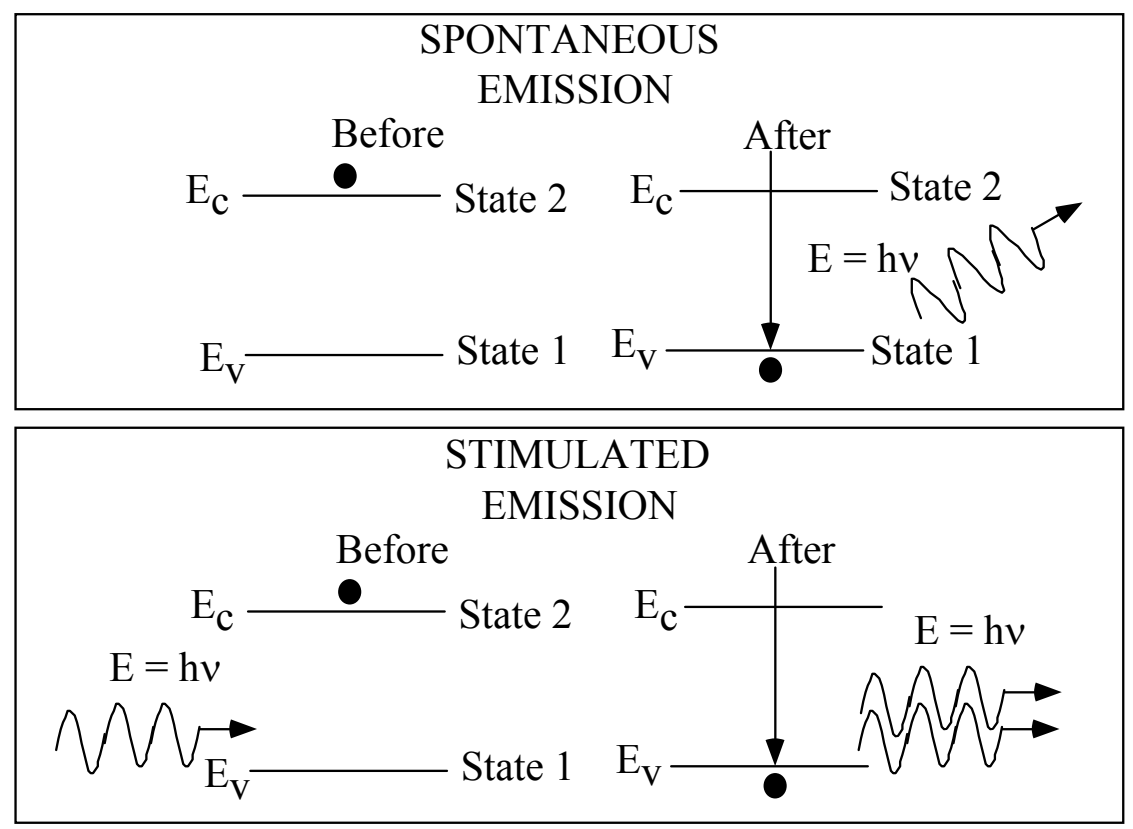

Fig. 1. Example of figure from lecture notes used in EEE 194 Optoelectronic Materials and Devices.

\section{Lecture Topics}

The topics explored in the course began with about three weeks on the fundamentals necessary to understand optoelectronic devices. Then each of the main optoelectronic devices was considered for about 2.5 weeks: photodetectors, light emitting diodes (LEDs), and laser diodes. The last two regular lectures focused on quantum wells. Optical communication was often used as an example of an application. The investigation of each optoelectronic device centered on its device physics, applications, and performance parameters. Emphasis was placed on choosing a specific device based on criteria such as wavelength of operation, cost, and performance. Discussions of the physical realizations of these devices were based on an understanding of crystal growth and device fabrication. One of the most difficult aspects of this course is the lack of a good textbook at the undergraduate level for currently changing topics such as white LEDs or vertical cavity surface emitting lasers (VCSELs). The required textbook was Singh's Optoelectronic Materials and Devices [5]. However, articles from sources such as Laser Focus World and IEEE Spectrum as well as instructor handouts were used extensively. Obtaining reference material at a level that is accessible to undergraduates is challenging but fun for the instructor.

Students at USD learn about basic semiconductor physics and the device physics of a pn junction/diode in their Engineering Materials Science class. Thus they need only a brief review of these topics. However, they also need to expand on these topics by considering 2-D band structure particularly as it relates to direct and indirect bandgap materials, semiconductor alloys particularly the importance of both lattice constant and bandgap, and high-field carrier motion including breakdown. Basic optical process (emission and absorption) are also new topics for them. A variety of processes are covered here including distinguishing between radiative and nonradiative emission, surface recombination, recombination lifetimes, quantifying absorption via an absorption coefficient, and bandedge, high energy, and low energy transitions. Students learn to distinguish between spontaneous and stimulated emission. (See Figure 1 for excerpt from lecture notes.) 
In the photodetector section, the general operation of converting optical energy to electrical energy is stressed. Then students are introduced using band diagrams and appropriate equations, to the physical operation of photodetectors including photodiodes, photoconductors, pin diodes, avalanche photodiodes, and phototransistors. Advantages of each type are considered. Key performance parameters of photodetectors are identified such as responsivity, efficiency, and spectral response. The materials issues involved in the design of photodetectors are investigated. For example, given appropriate information, students must be able to choose a suitable material for a window layer for a heterojunction photodiode and explain the purpose of a window layer as well as describe the importance of a constant gain-bandwidth product for photodetectors. In addition during this section, some time is spent discussing important devices whose operation is related to that of photodetectors including solar cells and charge coupled devices (CCDs). Noise is also considered since it is important in characterizing detector performance.

Given recent advances in LEDs, many changes were made in the LED section between the course offerings in 1999 and 2003 particularly on white LEDs. This section begins with the physical operation of LEDs and identification of performance parameters of LEDs such as efficiency and spectral response including distinguishing between injection efficiency, recombination efficiency, and extraction efficiency. Ways to maximize efficiency are considered including designing epoxy domes. The impact of the human eye for visible wavelengths is discussed including why $1 \mathrm{~mW}$ of light at different wavelengths would appear to have different brightness to a human. Key materials issues involved in the design of LEDs are explored including using direct versus indirect bandgap materials and identifying materials used for red, blue, and infrared LEDs. The current technology and applications of white LEDs are investigated and compared with conventional lighting. Viewgraphs and articles from the Lumileds website were helpful in this section [6]. Having simple physical examples of white, blue, red, and infrared LEDs wired to 9-V batteries were excellent pedagogical tools. The section concluded with some examples of LED applications including optoisolators and LEDs designed for telecommunications.

Lasers are the last major optoelectronic devices considered and students are usually excited to learn about their operation and the conditions necessary to achieve lasing. Population inversion is defined and students learn to explain how it is achieved in a laser diode. Performance parameters of lasers are identified such as efficiency and spectral response. The key materials issues involved in the design of laser diodes are explored. Students calculate the longitudinal mode spacing (in $\mathrm{nm}$ or $\mathrm{Hz}$ ) of a laser diode given the cavity length, operating wavelength, and refraction index of the material. They distinguish between optical and carrier confinement and explain how each is achieved in a laser diode. They learn to explain the concept of threshold for a laser and how to tell experimentally if a laser diode were operating above or below threshold and measure the threshold current. Several device designs that are used to lower threshold currents of laser diodes are considered. Distributed Bragg reflector (DBR) and distributed feedback (DFB) lasers are introduced. Students compare edge-emitting laser diodes and vertical cavity surface emitting laser diodes (VCSEL) including several advantages of each. Students learn to perform simple design calculations for DBR mirrors. Finally, a comparison between external and direct modulation for a laser diode is made.

The final topic in the course was an introduction to quantum wells. Quantum wells had already been mentioned in the discussion of laser diodes. Since undergraduates can easily become discouraged if presented with too many equations and may be intimidated by the words "quantum mechanics", the initial focus is to have them explain what a quantum well is, cite at least two reasons why quantum wells are interesting, and briefly describe the information that a wavefunction provides. The derivation of a simple equation for energy levels in a quantum well based on Schrödinger equation enables them to be able to estimate the energy levels (in $\mathrm{eV}$ ) of electrons or holes in a quantum well. The quantum confined stark effect (QCSE) in semiconductors and how it may be used in an electroabsorption modulator are also discussed.

\section{Laboratory}

The laboratory for this course was offered in the USD Optoelectronics Laboratory which has been supported by funding from the university as well as the National Science Foundation [7]. Facilities include two Newport optical tables, an Ocean Optics fiber-coupled spectrometer, a collection of optoelectronic positioners and mounts, the Newport Projects in Fiber Optics kit, an ILX Lightwave laser diode controller, an atomic force microscope, Newport optical power meters, electronic measuring equipment (multimeters, power supplies, pulse generators), and several computers with National Instruments LabVIEW hardware and software. Due to the expense of some of this equipment, having multiple stations is not possible. Since students learn better when they are active participants, most lab sessions included two groups of two students each and two lab exercises/stations. Thus it took two lab periods for the entire class to perform each experiment. Students wrote a formal group report following guidelines provided for most labs. For Experiments 4 and 7, individual reports specific to the experiment were required. 
Table 1. Experiments performed in Optoelectronic Materials and Devices in Spring 2003

\begin{tabular}{|c|l|}
\hline $\begin{array}{c}\text { Laboratory } \\
\text { Number }\end{array}$ & Laboratory Title \\
\hline 1 & Introduction to Fiber Optics \\
\hline 2 & Fiber Optics II : Transmitters, Receivers, and Cables \\
\hline 3 & Detectors of Light \\
\hline 4 & Introduction to Microfabrication \\
\hline 5 & Coupling Light into Multimode Fiber and Investigating Simple Optoelectronic Circuits \\
\hline 6 & Characterizing Sources of Light \\
\hline 7 & Optical Communication Links \\
\hline
\end{tabular}

The titles of the labs are listed in Table 1. Each lab will be described briefly focusing on its objectives. In Lab 1, students performed four modules as an Introduction to Fiber Optics: speed of light [8], total internal reflection, wave guiding, and optical voice link [9]. Students also read an article on the evolution of fiber optic cables [10]. This introductory exercise was intended to enable the students to explain wave guiding, describe total internal reflection and its importance for fiber optics, experiment with a simple fiber optic system, and calculate the speed of light using experimental data.

Lab 2 continued the investigation of Fiber Optics by having students take measurements of the attenuation of plastic fiber at several wavelengths and examining the optical voice link in more detail. Specifically, they learned to use a fiber-coupled spectrometer to measure the peak wavelength of an LED, use an optical power meter to measure the optical power emitted by an LED, calculate the absorption of a material at a given wavelength based on experimental data, observe the signals on an oscilloscope for an optical transmitter and receiver for a variety of input signals, and describe the main different in output signal between a fluorescent and incandescent light source.

In Lab 3, Detectors of Light using transmission measurements, students analyzed the absorption properties of several samples including one of their own choosing that they brought to lab such as sunglasses or film. Students also compared the response times of different detectors including a phototransistor, photodarlington, photoconductor, and photodiode [11].

The fourth lab, Introduction to Microfabrication Laboratory was intended to help students gain an appreciation for the semiconductor fabrication process that is also used to fabricate optoelectronic devices. Semiconductor fabrication is not covered in any other required course in the curriculum at USD. Students watched videos about the general process of semiconductor fabrication from crystal growth to computer assembly, "Silicon Run" and "Silicon Run II" [12], as well as a brief promotional CD showing modern $300 \mathrm{~mm}$ manufacturing with its automation [13]. They read the first two chapters of Jaeger's book on microelectronic fabrication [14]. The instructor also showed and discussed some physical examples including blank wafers, wafers with photoresist, fully processed wafers, bonded devices, layouts, and masks.

In one part of Lab 5, students coupled light from a HeNe laser into an optical fiber [15]. In the other part of Lab 5, they experimented with simple light and dark activated circuits, optoisolator circuits, and demonstrated their own night light. By the end of this lab, they were able to describe the issues involved in coupling light from a laser into an optical fiber, compare the response of circuits with photoconductors and phototransistors, and demonstrate the performance of an optoisolator. Although coupling light into a fiber is challenging and tedious, most students appreciated the opportunity to work with state of the art equipment.

In one part of Lab 6, Characterizing Sources of Light, students measured and compared optical power versus input electrical current (P-I curves) for a visible LED and a visible laser diode using LabVIEW. In the other part of Lab 6, students learned about different sources of light including white light sources (LED, W-Halogen), gas (HeNe) and diode lasers, and different color LEDs by comparing their emission spectra obtained using a fiber-coupled spectrometer.

In the last lab period, the students explored a fiber optic communication link and multiplexed two stereo signals. The sources of light were an LED and a laser diode and the analog modulation was provided by two tape players. Based on Project 8 of Newport's Projects in Fiber Optics Kit [16], this experiment was unique in that two students from the French Military Academy at St. Cyr had prepared the experimental set-up as part of their senior thesis. The French students also prepared and delivered a presentation to the USD students describing the operation of the link, the problems they had encountered, and their experimental results. They demonstrated the multiplexing and then the USD students did some experimentation of their own as shown in Figure 2. Due to the large number of components and the need for multiple precise alignments, it is not practical to have students construct the link entirely on their 
own in 1.5 hours. Having the link set up so that students see it working and then take some measurements allows them to focus on understanding rather than getting too frustrated by the alignments. The USD students were impressed with the French students' skill at using PowerPoint to animate graphics in their description of the multiplexer and felt it enhanced their own understanding of the technical content. After their own attempts to align light to fibers, they were especially impressed with the French students' experimental skill in assembling a complicated setup.

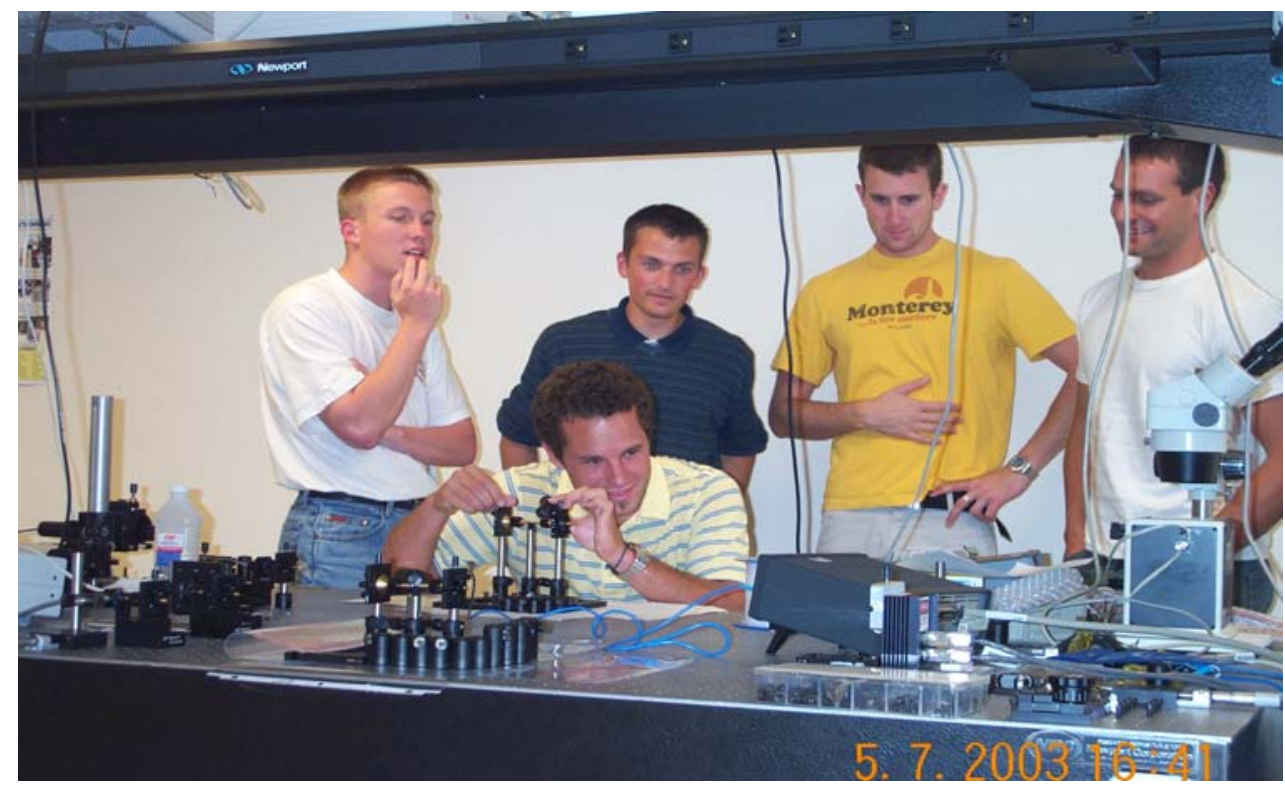

Fig. 2. Students experimenting with the optical communication link (Lab 7).

\section{Contemporary Issues in Optoelectronics}

Several innovative pedagogical techniques were integrated into the course. These brought contemporary optoelectronic issues into the classroom and allowed the students to develop some professional skills in research and communication. Engineers must demonstrate the ability to research a topic and communicate their findings orally and in writing. Thus, students were given the opportunity to explore an area more deeply by writing and presenting a paper on a topic of their choice. Topics ranged from organic LEDs to lasers in medicine to photonic crystals to free space communication systems. In the syllabus on the first day, the parameters of this project were described (presentation to class and report: 6-10 pages typed, cover sheet, appropriate documentation of multiple references). To enhance the quality of the final product in a busy semester, interim deadlines were provided. About four weeks into the class, students must submit a topic and state why they chose this topic, one thing they know about it, one thing they want to investigate, and give appropriate citations for at least 2 references. About four weeks later, they must submit an outline identifying the specific topics to be discussed. About three weeks later, a draft of the paper is due. It must be in the format of the final paper and is peer reviewed in class on the day it is submitted. Final papers are due two weeks later or about 1.5 weeks before the semester ends to allow time for the instructor to grade and return the papers on the last day of class. The final paper due date is also the first day of presentations. Each student presents for about 30 minutes including questions so two students present per class period. About one month before the end of the semester, students sign up for a presentation date and a consultation with the instructor a few days before their presentation date. Students peer review each other's presentation and the instructor provided a summary for each student including comments from peers and instructor and grades for content and presentation from peers and instructor. Most students did very well on the presentation with grades ranging from 75 to $100 \%$. The grading by the instructor and the students was quite consistent always being within $4 \%$ of each other. All students used PowerPoint. Some students included demonstrations, mini-lectures on the board, candy rewards, or interactive questions. Each presenter also prepared two questions with answers based on their presentation for possible use on the final exam. They were encouraged to share these with their classmates either during or after their presentation. After some editing by the instructor, a short question based on each presentation was included on the final exam. 
In Spring 2003, the last fifteen minutes of each Friday class was devoted to "Fabulous Friday" where one student led a discussion of a recent article which he/she had distributed to the class on Monday. The leader prepared a summary of the article and several questions for the class. Articles came from sources such as Laser Focus World and IEEE Spectrum. This endeavor gave the students an opportunity to develop oral communication skills and the ability to critically evaluate new information from sources other than textbooks and lectures. Topics included applications of LEDs for curing blindness, iris scanning for security, and thin-film photovoltaics. Some students used their Fabulous Friday topic as their paper topic. Some did outside research to enhance their discussions. Throughout the course of the semester, articles had been tending to get shorter until one very short one proved difficult to understand due to the lack of details provided. The class recognized this and discussed the criteria for a suitable article. Students enjoyed the range of topics. Leading a discussion was challenging for many including the instructor as she strives to balance her own participation, providing context or background, and letting the students lead.

Contemporary issues in optoelectronics were also incorporated into several homework assignments. For example, one homework assignment required students to evaluate an issue of Laser Focus World. They reviewed the "Back to Basics" article in their issue and identified the section that they found most interesting. Investigating the advertisements helped them learn about the diversity of current products and companies that make up the modern optoelectronic industry. It also forced them to look at a magazine that they might have initially found intimidating and helped to build up some confidence in their own ability to learn from such sources. In April 2003, the class visited the Navy's SPAWAR Systems Center and toured the electronic fabrication facilities as well as the labs in the Photonics and RF Branch. They learned about topics such as Bragg gratings, optical filtering, and fused fiber coupling and had the opportunity to talk with researchers about their careers.

\section{Student Response}

Student response to the class was quite enthusiastic as may be expected for a small elective course. Although students worked hard, they believed they learned a lot which made it worthwhile. At the end of the semester, students filled out separate evaluations for the lecture and laboratory. Each evaluation consisted of responding on a scantron to specific questions as well as written comments. The choices for student ratings were "Excellent, Very Good, Good, Fair, Poor, and Very Poor". All responses on both evaluations were Excellent, Very Good, or Good corresponding to median numerical ratings from 4.0 to 4.9. In the lecture, the highest ratings were reported (4.9, $88 \%$ Excellent, $12 \%$ Very Good) for "instructor's contribution to the course," "instructor's effectiveness in teaching the subject matter," "use of class time," and "course organization". The lowest rated category (4.0, 38\% Excellent, $25 \%$ Very Good, $38 \%$ Good) was "reasonableness of assigned work". In the laboratory, the highest rated category (4.9, 88\% Excellent, $12 \%$ Good) was "instructor's ability to deal with student difficulties." The lowest rated category (4.1, 25\% Excellent, 62\% Very Good, 12\% Good) was "content of the lab section". Given the nature of optoelectronic equipment, it is important that the instructor and students be flexible and adapt if things do not go as planned.

On the end of the semester evaluations for the lecture, all students reported that the class was intellectually challenging. The aspects of the class that they reported contributed most to their learning were the class handouts (5), active learning (3), labs (2), and Fabulous Fridays (2). Another student stated, "The focus on concepts with just enough math and lots of application was great. I didn't seem to get lost in the numbers." Several students commented that the book was too "intimidating" and could be improved. Four students said they had no suggestions for improving the class, one asked for more specifics on Fabulous Fridays, another for more emphasis on devices to balance out the materials section, another thought the paper and presentation were too heavily weighted ( $30 \%$ of class grade).

In the end of the semester evaluations for the laboratory, all students again reported that the lab was intellectually challenging. Responses to the aspect of this class that contributed most to learning included

- Open discussion labs and availability of extra help

- Lab write-ups because you have to think about the results you obtained to analyze them

- Being able to work in teams of two. Having lab every other week.

- The experiments were interesting and complimented the lecture material.

- Small class size and great lab equipment was a huge bonus. The field trip and presentations were beneficial. 
- Discussing the problems experienced in lab with lab instructor. This gave me a better understanding of the material covered in class and made me see some connections to real world applications.

- The class was well organized and spent the right amount of time on detectors and emitters. It was difficult to find time to work on the papers, so the numerous assignments for the paper really helped.

For aspects of the class that detracted from learning, 5 students said nothing. One wanted more lab time, another thought the paper was weighted too heavily and there was confusion about Fabulous Friday expectations, the third cited problems with a specific experiment that made analysis frustrating. For suggestions to improve the lab, five students indicated "nothing". One wished lecture were more like Fabulous Friday, another suggested a small experimental project at the end of the semester, and the third said "The lab portion was excellent. The only thing I wish we would have done was to build our own optical communication system, like the French students did."

An informal midcourse evaluation was also conducted. Based on some excellent suggestions, changes were made to the instructions on Fabulous Friday to include more details on the type of article that would be suitable (length, related to a topic discussed in class) and the requirements for the summary. Students were generally happy with the pace and structure of the course. Some wanted more examples and an effort was made to address this in subsequent lectures.

\section{Summary}

An undergraduate elective course on optoelectronic materials and devices is being developed at the University of San Diego. Lecture topics include semiconductor fundamentals as well as essential optoelectronic devices: LEDs, lasers, and photodetectors. Students performed seven laboratory experiments, chose a topic related to optoelectronics, wrote a paper, and delivered a presentation to the class. Further opportunities to explore current topics and improve presentation skills were provided in homework assignments and "Fabulous Friday" discussions. Student response to the class as demonstrated by course evaluations was very positive indicating that they found it challenging but worthwhile.

\section{Acknowledgments}

The author would like to thank the students in USD EEE 194 in Spring 1999 and 2003. She is also grateful to Dr. F. Assaderaghi for obtaining the TSMC CD, G. Frocklage for the LED physical demos, D. M. Tawy, S. D. Alsaialy, and M. L. Higa for assistance with LabVIEW, and B. Malbrancke and S. Drenne for the final laboratory on optical communication links.

\section{References}

[1] W. T. Cathey, Guest Editorial, IEEE Transactions on Education, 35, 101 (1992).

[2] IEEE Transactions on Education, 35, (1992).

[3] See, for example, The 1995 International Conference on Education in Optics, Proc. SPIE Conf. Education in Optics, vol. $2525,1995$. ETOP' 97 Fifth Topical Meeting on Education and Training on Optics and Photonics, Delft, The Netherlands, August 1997. Proc. SPIE Conf. Education in Optics, vol. 3190, 1997.

ETOP' 99 Sixth Topical Meeting on Education and Training on Optics and Photonics, Cancun, Mexico, July 1999. Proc. SPIE Conf. Education in Optics, vol. 3831, 1999. http://www2.uaem.mx/cii/etop.html

[4] R. Felder, "Reaching the Second Tier: Learning and Teaching Styles in College Science Education." J. College Science Teaching, 23(5), 286290 (1993).

[5] Jasprit Singh, Optoelectronics: An Introduction to Materials and Devices, (McGraw-Hill, New York, 1996). Note that this book is out of print. Permission to reproduce copies for the students was obtained from the publisher.

[6] http://www.lumileds.com

[7] "Optoelectronic Materials and Device Characterization" National Science Foundation CAREER Program, ECS-9796220.

"An Optoelectronics Laboratory for Undergraduates" National Science Foundation Instrumentation and Laboratory Improvement (ILI) Program, DUE-9796201.

[8] Speed of Light Apparatus, Industrial Fiber Optics part IF-SL-K (assembled) http://www.i-fiberoptics.com/educ/fomodul.htm

[9] S. M. Lord, "Optoelectronics Experiments for First Year Engineering Students," IEEE Transactions on Education, 44, 16-23 (2001).

[10] Ira C. Magaziner and Mark Patinkin, "Corning Glass: The Battle to Talk with Light" in The Silent War, (Random House, New York, 1989).

[11] Based on Activity VII from Fiber Optic Lab Manual, Industrial Fiber Optics, $3^{\text {rd }}$ edition, 1998.

[12] Ruth Carranza Productions, Mountain View, California, 1990 and 1993. http://www.siliconrun.com/

[13] "Voyage into the Future: TSMC's FAB 12 and $300 \mathrm{~mm}$ Manufacturing," TSMC, 2002.

[14] R. C. Jaeger, Introduction to Microelectronic Fabrication, (Addison-Wesley, Boston, 1988).

[15] C. J. Theesfeld and S. M. Lord, "Designing Optoelectronic Laboratories: A Unique Senior Design Opportunity," in Proceedings of the 1996 Frontiers in Education Conference, Salt Lake City, Utah, (1996).

[16] Projects in Fiber Optics Kit, Newport Corporation, part FKP-STD-OD http://www.newport.com . 